\title{
Clinical features of critically ill patients infected with SARS-CoV-2 outside Wuhan with and without diabetes
}

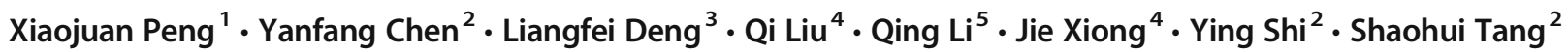

Received: 24 August 2020 / Accepted: 21 October 2020 / Published online: 5 November 2020

(C) Research Society for Study of Diabetes in India 2020

\begin{abstract}
Aim Some patients with severe acute respiratory syndrome coronavirus 2 (SARS-CoV-2) infection rapidly develop to critical condition. Here, we investigated the clinical features of critically ill SARS-CoV-2 patients with and without diabetes and identified risk factors for death of these patients.

Methods The medical records including epidemiological, demographic, clinical, and laboratory data from 49 critically ill SARSCoV-2 patients were collected and analyzed in Huanggang City and Xiaogan City, Hubei Province, outside Wuhan.

Results Sixty-seven percent (33) of patients survived and 33\% (16) of patients died in 49 critically ill patients (32 men, 17 women), with a median age of 63 years (IQR 53-73). Univariate analyses indicated that the deceased patients were more often associated with two or more comorbidities, one or more gastrointestinal symptoms, high neutrophil percentage, low lymphocytes and lymphocyte percentage, high C-reactive protein, high procalcitonin, high fasting blood glucose (FBG), and high lactate dehydrogenase (LDH) compared with the survivors; moreover, the patients with T2DM had the higher neutrophil percentage, the lower lymphocyte percentage, and the higher levels of FBG and LDH compared with the patients without T2DM. Multivariable logistic regression analyses indicated that gastrointestinal symptoms ( $\geq 1$ symptoms), decreased lymphocytes $\left(<1.1 \times 10^{9} / \mathrm{L}\right)$, and increased FBG $(\geq 7.0 \mathrm{mmol} / \mathrm{L})$ were the independent risk factors for death of critically ill patients.

Conclusions Critically ill COVID patients with T2DM had more severe damages of the lymphocytes, islet cells, and heart function, and gastrointestinal symptoms, lymphopenia, and increased FBG may be early predictors for poor prognosis.
\end{abstract}

Keywords SARS-CoV-2 $\cdot$ Critically ill patients $\cdot$ Clinical features $\cdot$ T2DM $\cdot$ Independent risk factors

\section{Introduction}

Coronaviruses (CoVs) are enveloped, positive-sense, singlestranded RNA viruses and belong to the family Coronaviridae and the order Nidovirales, of which two coronaviruses, severe acute respiratory syndrome coronavirus (SARS-CoV) $[1,2]$ and Middle East respiratory syndrome coronavirus (MERS$\mathrm{CoV}$ ) [3], have caused recent pandemics of respiratory

Xiaojuan Peng, Yanfang Chen, Liangfei Deng, Qi Liu and Qing Li contributed equally to this work.

Supplementary Information The online version contains supplementary material available at https://doi.org/10.1007/s13410-02000888-3.

Shaohui Tang

tangshaohui206@163.com

1 Department of Endocrinology, Affiliated Hospital (Clinical College) of Xiangnan University, Chenzhou 423000, Hunan, People's Republic of China

2 Department of Gastroenterology, The First Affiliated Hospital, Jinan University, Guangzhou 510630, Guangdong, People's Republic of China
3 Department of Critical Medicine, Affiliated Hospital (Clinical College) of Xiangnan University, Chenzhou 423000, Hunan, People's Republic of China

4 Department of Infectious Diseases, the First People's Hospital of Xiaochang County, Xiaogan 432900, Hubei, People's Republic of China

5 Department of Interventional Vascular Surgery, Affiliated Hospital (Clinical College) of Xiangnan University,

Chenzhou 423000, Hunan, People's Republic of China 
infectious diseases with high mortality rates of $10 \%$ for SARS-CoV [4] and 37\% for MERS-CoV [5].

On Jan 7, 2020, a novel coronavirus was identified by the Chinese Center for Disease Control and Prevention (CDC) from the lower respiratory tract sample of a patient, and subsequently named severe acute respiratory syndrome coronavirus 2 (SARS-CoV-2) by the World Health Organization (WHO) [6]. Since the first case was reported in December 2019 in Wuhan, China, the outbreak of the disease is currently continuously spreading all over the world.

The disease caused by SARS-CoV-2 that is named as coronavirus disease 2019 (COVID-19) by the WHO could induce symptoms including fever, dry, cough, dyspnea, fatigue, and lymphopenia in infected patients, with some patients rapidly developing acute respiratory distress syndrome (ARDS), acute respiratory failure, or multiple organ failure and even death [7]. Mechanical ventilation is usually required in critically ill patients with ARDS or acute respiratory failure. At present, although many articles have established the epidemiology and clinical features of patients with SARS-CoV-2 infection [7-10], the information regarding clinical manifestations and laboratory findings in critically ill patients with COVID-19 has not been reported yet. In the present study, we investigated the clinical features of critically ill patients with COVID-19 and identified the independent risk factors for death of these patients in Huanggang City and Xiaogan City, Hubei Province, outside Wuhan.

\section{Methods}

\section{Patients}

We retrospectively analyzed the medical records from critically ill patients with COVID-19 admitted to Dabie Mountain Medical Center and the First People's Hospital of Xiaochang County, Hubei Province, from February 1 to March 25, 2020. The two hospitals, which are located in Huanggang City and Xiaogan City, respectively, Hubei Province, about $80-90 \mathrm{~km}$ away from Wuhan, China, are the designated hospitals for the hospitalization of patients with COVID-19. SARS-CoV-2 pneumonia was diagnosed based on clinical symptoms with typical changes in chest $\mathrm{CT}$ and positive for the nucleic acids of SARS-CoV-2. Severity of COVID-19 was defined according to the diagnostic and treatment guideline for SARS-CoV-2 issued by the Chinese National Health Committee (version 3-6). Critically ill patients with COVID-19 were designated when the patients had one of the following criteria: (a) respiratory failure with mechanical ventilation; (b) shock; (c) combination with other organ failures, with ICU monitoring and treatment.

\section{Data collection}

Epidemiological, demographic, clinical, laboratory, and medical imaging data from patients' medical records were collected by Qi Liu and Liangfei Deng who is a doctor of critical medicine and rush to the rescue of Dabie Mountain Medical Center on February 1, 2020. If data were missing from the records or clarification was needed, we obtained data by direct communication with attending doctors and patients or their families. The nucleic acid testing for SARS-CoV-2 was performed using quantitative RT-PCR on samples from the respiratory tract by Huanggang CDC and Xiaogan CDC, which are the designated laboratory for SARS-CoV-2 test.

\section{Statistical analysis}

Categorical data were expressed as number (\%) and evaluated by $\chi 2$ or Fisher's exact test; continuous data were expressed as median (interquartile range (IQR)) and evaluated by MannWhitney $U$ test. To explore the risk factors associated with death of critically ill patients, a multivariable logistic regression model was used. A two-sided $\alpha$ of less than 0.05 was considered statistically significant. All the statistical analyses were performed with SPSS (version 26.0).

\section{Results}

\section{Demographics and clinical characteristics}

A total of 49 critically ill patients with COVID-19, who all were admitted to intensive care unit (ICU) and required oxygen therapy, were included in this study (32 men, 17 women), with a median age of 63 years (IQR 53-73). Thirty-three patients were discharged and 16 patients were deceased. Among them, there were 26 patients with familiar/cluster exposure history and 23 cases with community exposure history. Seventy-three percent of patients had comorbid chronic systemic diseases, including hypertension (45\%), chronic heart disease (33\%), type 2 diabetes mellitus (T2DM) (22\%), chronic obstructive pulmonary disease $(22 \%)$, cerebrovascular disease (18\%), chronic liver disease (10\%), chronic renal disease (4\%), and malignant tumor (4\%), of whom $57 \%$ of patients had two or more of the comorbidities. The median intervals from disease onset to admission and from admission to critical condition were 7.0 days (IQR 4.5-10.0) and 4.0 days (IQR 2.5-8.0), respectively. The deceased patients were more often associated with two or more of comorbidities and shorter time from admission to critical condition compared with the survivors, whereas there were no significant differences between the survivors and deceased groups with respect to age, sex, exposure history, occupation, smokers, etc. (Table 1). 
Table 1 Demographics and baseline characteristics of critically ill patients with COVID-19

\begin{tabular}{|c|c|c|c|c|}
\hline Items & All patients $(n=49)$ & Survivors $(n=33)$ & Deceased $(n=16)$ & $p$ value \\
\hline Age, years & $63(53-73)$ & $58(50-74)$ & $67(60-72)$ & 0.267 \\
\hline \multicolumn{5}{|l|}{ Sex, $n(\%)$} \\
\hline Female & $17(35)$ & $12(36)$ & $5(31)$ & \multirow[t]{2}{*}{0.724} \\
\hline Male & $32(65)$ & $21(64)$ & $11(69)$ & \\
\hline \multicolumn{5}{|l|}{ Exposure history, $n(\%)$} \\
\hline Familiar/cluster infections & $26(53)$ & $16(48)$ & $10(63)$ & 0.357 \\
\hline Community infections & $23(47)$ & $17(52)$ & $6(37)$ & 0.357 \\
\hline \multicolumn{5}{|l|}{ Occupation, $n(\%)$} \\
\hline Agricultural worker & $13(27)$ & $9(27)$ & $4(25)$ & 0.867 \\
\hline Employee & $11(22)$ & $8(24)$ & $3(19)$ & 0.669 \\
\hline Retired & $25(51)$ & $16(49)$ & $9(56)$ & 0.610 \\
\hline Smokers, $n(\%)$ & $18(37)$ & $11(33)$ & $7(44)$ & 0.478 \\
\hline Chronic systemic diseases, $n(\%)$ & $36(73)$ & $22(67)$ & $14(88)$ & 0.174 \\
\hline Hypertension & $22(45)$ & $13(39)$ & $9(56)$ & 0.266 \\
\hline Chronic heart disease & $16(33)$ & $10(30)$ & $6(38)$ & 0.614 \\
\hline $\mathrm{T} 2 \mathrm{DM}$ & $11(22)$ & $6(18)$ & $5(31)$ & 0.456 \\
\hline Chronic obstructive pulmonary disease & $11(22)$ & $8(24)$ & $3(19)$ & 1.000 \\
\hline Cerebrovascular disease & $9(18)$ & $4(12)$ & $5(31)$ & 0.130 \\
\hline Chronic liver disease & $5(10)$ & $4(12)$ & $1(6)$ & 1.000 \\
\hline Chronic renal disease & $2(4)$ & $1(3)$ & $1(6)$ & 1.000 \\
\hline Malignancy & $2(4)$ & $1(3)$ & $1(6)$ & 1.000 \\
\hline Two or more of the above diseases & $28(57)$ & $15(45)$ & $13(81)$ & 0.018 \\
\hline Days from disease onset to admission, days & $7.0(4.5-10.0)$ & $6.0(4.0-10.0)$ & $8.0(6.3-10.8)$ & 0.534 \\
\hline Days from admission to critical condition, days & $4.0(2.5-8.0)$ & $6.0(3.5-8.5)$ & $2.5(1.0-4.0)$ & 0.001 \\
\hline
\end{tabular}

Data are shown as median (IQR) or $n(\%)$. $p$ values comparing survivors and deceased are from $\chi^{2}$ test, Fisher's exact test, or Mann-Whitney $U$ test. COVID-19, coronavirus disease 2019; IQR, interquartile range

The most common symptoms at disease onset were fever $(82 \%)$, cough $(76 \%)$, fatigue $(57 \%)$, gastrointestinal symptoms $(47 \%)$, and chest tightness/dyspnea (45\%). Gastrointestinal symptoms included nausea or vomiting $(31 \%)$, diarrhea $(22 \%)$, anorexia (16\%), and abdominal pain $(6 \%)$. The gastrointestinal symptoms were more common in the deceased patients compared with the survivors, whereas there were no significant differences between the survivors and deceased groups about the other symptoms (Table 2).

\section{Laboratory and imaging findings}

On admission, leucocytes were within the normal range in most of the patients (59\%), with $35 \%$ increased and $6 \%$ decreased numbers. Neutrophils were above the normal range in $39 \%$ of patients, and nearly half of the patients $(47 \%)$ had increased neutrophil percentage. Lymphopenia was common, and lymphocytes and lymphocyte percentage were below the normal range in $71 \%$ and $65 \%$ of the patients, respectively. Platelets and hemoglobin were below the normal range in $20 \%$ of patients and $45 \%$ of patients, respectively. Most of the patients had increased levels of C-reactive protein (CRP) (86\% cases) and procalcitonin (PCT) (55\% cases), with a median CRP level of $37.4 \mathrm{mg} / \mathrm{L}$ (IQR 16.0-62.4) and median PCT level of $0.6 \mathrm{ng} / \mathrm{mL}$ (IQR 0.1-3.8) (Table 3).

On admission, most patients showed normal prothrombin time, and all patients showed normal activated partial thromboplastin time. D-dimer level was above the normal range in $59 \%$ of patients, with a median D-dimer level of $0.6 \mu \mathrm{g} / \mathrm{mL}$ (IQR 0.2-1.3) (Table 3).

The median fasting blood glucose (FBG) level of the patients was $6.9 \mathrm{mmol} / \mathrm{L}$ (IQR 5.7-9.7), with $\mathrm{FBG} \geq 7.0 \mathrm{mmol} / \mathrm{L}$ in $49 \%$ of patients on admission. The patients had liver function abnormality, with $57 \%$ decreased albumin level, $8 \%$ increased alanine aminotransferase (ALT) or $14 \%$ increased aspartate aminotransferase (AST) level, and 22\% increased total bilirubin level. The patients had abnormal myocardial zymogram, which showed the elevation of creatine kinase (CK) in $31 \%$ of patients, the elevation of MB isoenzyme of creatine kinase (CKMB) in $16 \%$ of patients, and the elevation of lactate dehydrogenase (LDH) in $76 \%$ of patients. The patients had renal function damage, with elevated blood urea nitrogen 
Table 2 Clinical manifestations of critically ill patients with 2019-nCoV pneumonia

\begin{tabular}{|c|c|c|c|c|}
\hline Items & All patients $(n=49)$ & Survivors $(n=33)$ & Deceased $(n=16)$ & $p$ value \\
\hline Fever, $n(\%)$ & $40(82)$ & $28(85)$ & $12(75)$ & 0.449 \\
\hline Cough, $n(\%)$ & $39(80)$ & $27(82)$ & $12(75)$ & 0.709 \\
\hline Fatigue, $n(\%)$ & $28(57)$ & $21(64)$ & $7(44)$ & 0.187 \\
\hline Muscle ache, $n(\%)$ & $9(18)$ & $5(15)$ & $4(25)$ & 0.449 \\
\hline Headache, $n(\%)$ & $5(10)$ & $2(6)$ & $3(19)$ & 0.313 \\
\hline Sore throat, $n(\%) \mathrm{t}$ & $4(8)$ & $2(6)$ & $2(13)$ & 0.588 \\
\hline Chill, $n(\%)$ & $3(6)$ & $1(3)$ & $2(13)$ & 0.245 \\
\hline Chest tightness/dyspnea, $n(\%)$ & $22(45)$ & $13(39)$ & $9(56)$ & 0.266 \\
\hline Gastrointestinal symptoms, $n(\%)$ & $23(47)$ & $10(30)$ & $12(75)$ & 0.003 \\
\hline Nausea or vomiting & $15(31)$ & $8(24)$ & $7(44)$ & 0.198 \\
\hline Diarrhea & $11(22)$ & $5(15)$ & $6(38)$ & 0.141 \\
\hline Anorexia & $8(16)$ & $3(9)$ & $5(31)$ & 0.094 \\
\hline Abdominal pain & $3(6)$ & $1(3)$ & $2(13)$ & 0.245 \\
\hline
\end{tabular}

Data are shown as $n(\%) . p$ values comparing survivors and deceased are from $\chi^{2}$ test or Fisher's exact test. COVID-19, coronavirus disease 2019; IQR, interquartile range

in $22 \%$ of patients and elevated serum creatinine in $22 \%$ of patients (Table 3).

On admission, all patients showed bilateral viral pneumonia in chest $\mathrm{CT}$ images. The representative chest $\mathrm{CT}$ findings of a deceased patient and a discharged patient showed bilateral ground glass opacity (Table 3; Supplementary Fig. 1).

The deceased patients had higher median neutrophil percentage, lower median lymphocytes and lymphocyte percentage, higher median C-reactive protein level and procalcitonin level, higher median fasting blood glucose level, and higher median lactate dehydrogenase level compared with the survivors, whereas there were no significant differences between the survivors and deceased groups concerning other blood routine, coagulation function, and other blood biochemistry parameters (Table 3).

\section{Treatment regimen}

According to the diagnostic and treatment guideline for COVID-19 issued by the Chinese National Health Committee (version 3-6), all patients received antiviral therapy, including combination of interferon- $\alpha$ ( 5 million $U$, twice daily, inhalationally) and lopinavir/ritonavir tablets $(500 \mathrm{mg}$, twice daily, orally) or combination of interferon- $\alpha$ ( 5 million $\mathrm{U}$, twice daily, inhalationally) and abidol (200 mg, three times daily, orally). The duration of treatment was 4-10 days. Sixtyone percent of patients were given corticosteroid treatment (methylprednisolone or dexamethasone) for 4-7 days. Fortynine percent of patients were administered with empirical antibiotic treatment (cephalosporins, quinolones, or carbapenems) for 5-12 days. Four (8.2\%) patients were also treated with antifungal drugs (voriconazole) (Supplementary Table 1).

All patients received respiratory support for 5-18 days, with $45 \%$ non-invasive ventilation and $55 \%$ invasive ventilation, of whom a patient was given combination of invasive ventilation and extracorporeal membrane oxygenation (ECMO). Four patients were treated with continuous renal replacement therapy (CRRT) in deceased patients. Moreover, $90 \%$ of patients were administered with traditional Chinese medicine (Lianhua Qingwen granules or capsules). There were no significant differences between the survivors and deceased groups about the above treatment regimens except for CRRT (Supplementary Table 1).

\section{Demographics and clinical and laboratory characteristics of 11 critically ill COVID patients with T2DM}

In 49 critically ill patients with COVID-19, 11 patients had history of T2DM (7 men, 4 women), with a median age of 58 years (IQR 51-62). The median duration of T2DM and the median $\mathrm{HbA} 1 \mathrm{c}$ level of the patients were 15 years and $8.2 \%$, respectively. All 11 patients had complications, including diabetic peripheral neuropathy $(64 \%)$, diabetic nephropathy $(36 \%)$, diabetic retinopathy $(36 \%)$, and diabetic macroangiopathy ( $9 \%$ ), of whom $36 \%$ of patients had two or more of the above complications. They received medications including sulfonylureas, biguanide, alpha-glucosidase inhibitors, glinides, DPP-IV inhibitors, and long-acting insulin analogs (Table 4). We compared the demographics and laboratory characteristics between 11 diabetic and 38 nondiabetic critically ill COVID patients according to the laboratory 
Table 3 Laboratory characteristics of critically ill patients with COVID-19

\begin{tabular}{|c|c|c|c|c|}
\hline Items & All patients $(n=49)$ & Survivors $(n=33)$ & Deceased $(n=16)$ & $p$ value \\
\hline \multicolumn{5}{|l|}{ Blood routine } \\
\hline Leucocytes $\left(\times 10^{9} / \mathrm{L}\right.$; normal range $\left.3.5-9.8\right)$ & $5.8(4.1-10.1)$ & $5.5(4.2-9.8)$ & $10.3(3.7-14.7)$ & 0.277 \\
\hline Increased, $n(\%)$ & $17(35)$ & $9(27)$ & $8(50)$ & 0.117 \\
\hline Decreased, $n(\%)$ & $3(6)$ & $2(6)$ & $1(6)$ & 1.000 \\
\hline Neutrophils $(\times 109 / \mathrm{L}$; normal range $1.8-6.3)$ & $4.1(2.8-9.5)$ & $3.9(2.7-6.0)$ & $9.5(3.0-13.5)$ & 0.050 \\
\hline Increased, $n(\%)$ & $19(39)$ & $10(30)$ & $9(56)$ & 0.08 \\
\hline Neutrophil percentage, $(\%)$ (normal range $40-75$ ) & $74.6(65.6-87.6)$ & $70.3(61.4-78.7)$ & $88.8(82.3-93.0)$ & 0.000 \\
\hline Increased, $n(\%)$ & $23(47)$ & $10(30)$ & $13(81)$ & 0.001 \\
\hline Lymphocytes $\left(\times 10^{9} / \mathrm{L}\right.$; normal range $\left.1.1-3.2\right)$ & $0.8(0.6-1.4)$ & $1.0(0.7-1.6)$ & $0.6(0.4-0.8)$ & 0.002 \\
\hline Decreased, $n(\%)$ & $35(71)$ & $20(61)$ & $15(94)$ & 0.019 \\
\hline Lymphocyte percentage, (\%) (normal range 20-50) & $15.6(8.7-25.1)$ & $19.8(11.3-26.6)$ & $8.7(3.4-11.7)$ & 0.000 \\
\hline Decreased, $n(\%)$ & $32(65)$ & $17(52)$ & $15(94)$ & 0.004 \\
\hline Platelets $\left(\times 10^{9} / \mathrm{L}\right.$; normal range $\left.125-350\right)$ & $178(131-226)$ & $130(115-145)$ & $147(120-201)$ & 0.150 \\
\hline Decreased, $n(\%)$ & $10(20)$ & $6(18)$ & $4(25)$ & 0.709 \\
\hline Hemoglobin (normal range $115-150 \mathrm{~g} / \mathrm{L}$ ) & $131(115-145)$ & $130(115-145)$ & $134(113-146)$ & 0.991 \\
\hline Decreased, $n(\%)$ & $22(45)$ & $16(48)$ & $6(38)$ & 0.468 \\
\hline \multicolumn{5}{|l|}{ Infection biomarkers } \\
\hline C-reactive protein $(\mathrm{mg} / \mathrm{L}$; normal range $0.0-8.0)$ & $37.4(16.0-62.4)$ & $31.2(10.0-56.0)$ & $58.3(26.7-90.0)$ & 0.047 \\
\hline Increased, $n(\%)$ & $42(86)$ & $27(82)$ & $15(94)$ & 0.402 \\
\hline Procalcitonin $(\mathrm{ng} / \mathrm{mL}$; normal range $0.0-0.5)$ & $0.6(0.1-3.8)$ & $0.2(0.1-1.2)$ & $3.0(0.6-7.5)$ & 0.004 \\
\hline Increased, $n(\%)$ & $27(55)$ & $14(42)$ & $13(81)$ & 0.010 \\
\hline \multicolumn{5}{|l|}{ Coagulation function } \\
\hline Prothrombin time (s; normal range 9.0-15.0) & $13.2(12.2-14.2)$ & $13.2(11.8-13.9)$ & $13.7(13.0-14.5)$ & 0.088 \\
\hline Increased, $n(\%)$ & $7(14)$ & $4(12)$ & $3(19)$ & 0.668 \\
\hline Activated partial thromboplastin time (s; normal range $22.0-45.0$ ) & $30.3(28.2-33.0)$ & $30.3(28.6-32.4)$ & $31.2(26.1-35.6)$ & 0.749 \\
\hline D-dimer $(\mu \mathrm{g} / \mathrm{mL}$; normal range $0.0-0.5)$ & $0.6(0.2-1.3)$ & $0.6(0.2-1.1)$ & $0.8(0.1-2.2)$ & 0.654 \\
\hline Increased, $n(\%)$ & $29(59)$ & $19(58)$ & $10(63)$ & 0.742 \\
\hline \multicolumn{5}{|l|}{ Blood biochemistry } \\
\hline Fasting blood glucose $(\mathrm{mmol} / \mathrm{L}$; normal range 3.9-6.1) & $6.9(5.7-9.7)$ & $6.1(5.3-7.9)$ & $8.3(7.1-12.7)$ & 0.003 \\
\hline Increased $(\geq 7.0 \mathrm{mmol} / \mathrm{L}), n(\%)$ & $24(49)$ & $11(33)$ & $13(81)$ & 0.002 \\
\hline Albumin $(\mathrm{g} / \mathrm{L} ;$ normal range $35.0-52.0)$ & $34.1(29.5-37.7)$ & $34.7(29.2-39.2)$ & $33.7(29.8-36.8)$ & 0.587 \\
\hline Decreased, $n(\%)$ & $28(57)$ & $17(52)$ & $11(69)$ & 0.253 \\
\hline Alanine aminotransferase (U/L; normal range 9-50) & $20.0(14.5-33.0)$ & $19.0(1.5-32.5)$ & $21.5(14.0-36.8)$ & 0.579 \\
\hline Increased, $n(\%)$ & $4(8)$ & $3(9)$ & $1(6)$ & 1.000 \\
\hline Aspartate aminotransferase (U/L; normal range $15-40)$ & $23.0(1.08-34.9)$ & $22.0(18.0-34.5)$ & $26.5(17.7-35.3)$ & 0.685 \\
\hline Increased, $n(\%)$ & $7(14)$ & $5(15)$ & $2(13)$ & 1.000 \\
\hline Total bilirubin $(\mu \mathrm{mol} / \mathrm{L}$; normal range $5.1-23.0)$ & $14.4(9.3-22.1)$ & $13.5(8.8-19.0)$ & $15.2(10.8-32.8)$ & 0.365 \\
\hline Increased, $n(\%)$ & $11(22)$ & $5(15)$ & $6(38)$ & 0.141 \\
\hline Creatine kinase (U/L; normal range 26-174) & $113.0(58.5-196.0)$ & $108.0(58.5-191.0)$ & $118.5(58.3-225.8)$ & 0.550 \\
\hline Increased, $n(\%)$ & $15(31)$ & $10(30)$ & $5(31)$ & 0.946 \\
\hline Creatine kinase-MB (U/L; normal range 3-25) & $13.5(9.0-23.2)$ & $12.1(8.4-27.0)$ & $16.4(10.1-22.5)$ & 0.354 \\
\hline Increased, $n(\%)$ & $8(16)$ & $5(15)$ & $3(19)$ & 1.000 \\
\hline Lactate dehydrogenase (U/L; normal range 109-245) & $315.6(216.5-449.0)$ & $268.6(201.6-367.6)$ & $443.4(279.7-528.5)$ & 0.016 \\
\hline Increased, $n(\%)$ & $37(76)$ & $23(70)$ & $14(88)$ & 0.290 \\
\hline Serum creatinine $(\mu \mathrm{mol} / \mathrm{L}$; normal range $32-106)$ & $69.5(61.0-96.4)$ & $68.9(57.7-88.0)$ & $86.6(64.3-112.5)$ & 0.147 \\
\hline Increased, $n(\%)$ & $11(22)$ & $6(18)$ & $5(31)$ & 0.466 \\
\hline Blood urea nitrogen (mmol/L; normal range 1.5-7.5) & $5.5(4.4-7.1)$ & $5.5(4.4-7.1)$ & $5.4(4.5-9.7)$ & 0.815 \\
\hline Increased, $n(\%)$ & $11(22)$ & $7(21)$ & $4(25)$ & 1.000 \\
\hline Bilateral involvement of chest $\mathrm{CT}$ images, $n(\%)$ & $49(100)$ & $33(100)$ & $16(100)$ & - \\
\hline
\end{tabular}

Data are shown as median (IQR) or $n(\%)$. $p$ values comparing survivors and deceased are from $\chi^{2}$ test, Fisher's exact test, or Mann-Whitney $U$ test. COVID-19, coronavirus disease 2019; IQR, interquartile range

parameters which showed significant differences between the survivors and deceased groups. The result indicated the patients with T2DM had the higher neutrophil percentage, the lower lymphocyte percentage, and the higher levels of FBG and LDH compared with the patients without T2DM; but there were no significant differences between the diabetic and nondiabetic patients concerning age, sex, infection biomarkers, and mortality rate (Table 5).

\section{The independent risk factors for poor prognosis}

To investigate the risk factors for poor prognosis in our cohort of 49 critically ill patients with SARS-CoV-2 infection, we performed a multivariable logistic regression analysis by the forward method using clinical manifestations and laboratory parameters which showed significant differences between the survivors and deceased groups. The result indicated that 
gastrointestinal symptoms, decreased lymphocytes, and increased fasting blood glucose were the independent risk factors for death of critically ill patients (Table 6).

\section{Discussion}

According to the diagnostic and treatment guideline for COVID-19 issued by the Chinese National Health Committee (version 3-6) (http://www.nhc.gov.cn/), the clinical classification of severity of COVID-19 includes four types: mild, common, severe, and critical. In this study, we reported clinical features of 49 critically ill patients with COVID-19 confirmed by clinical and laboratory results, who needed mechanical ventilation therapy in ICU. These patients came from Huanggang City and Xiaogan City,

Table 4 Demographics and baseline data of 11 critically ill COVID patients with T2DM

\begin{tabular}{ll}
\hline Items & Values \\
\hline Age, years & $58(51-62)$ \\
Sex, $n(\%)$ & \\
Female & $4(36)$ \\
Male & $7(64)$ \\
Duration of T2DM, years & $15.0(11.0-20.0)$ \\
HbA1c, $\%$ & $8.2(7.6-8.8)$ \\
Complications, $n$ (\%) & $11(100)$ \\
Diabetic peripheral neuropathy & $7(64)$ \\
Diabetic nephropathy & $4(36)$ \\
Diabetic retinopathy & $4(36)$ \\
Diabetic macroangiopathy & $1(9)$ \\
Two or more of the above complications & $4(36)$ \\
Treatment regimen, $n$ (\%) & $2(18)$ \\
Sulfonylureas & $1(9)$ \\
Biguanide & $1(9)$ \\
Alpha-glucosidase inhibitors & $1(9)$ \\
Combination of sulfonylureas and biguanide & $1(9)$ \\
Combination of long-acting insulin analogs and & $1(9)$ \\
biguanide & \\
Combination of alpha-glucosidase inhibitors and & $2(18)$ \\
bombinide & $1(9)$ \\
glinides & $1(9)$ \\
Combination of biguanide and glinides & \\
\hline
\end{tabular}

Data are shown as $n(\%)$ or median (IQR). COVID-19, coronavirus disease 2019; $I Q R$, interquartile range; $T 2 D M$, type 2 diabetes mellitus. Sulfonylureas: glimepiride ( $2 \mathrm{mg}$ qd po) or gliclazide ( $80 \mathrm{mg}$ bid po); biguanide: metformin ( $0.5 \mathrm{~g}$ bid po); alpha-glucosidase inhibitors: acarbose (50 mg tid po); glinides: repaglinide (1 mg tid po); DPP-IV inhibitors: sitagliptin (100 mg qd po); long-acting insulin analogs: lantus (16 U IH)
Hubei Province, 80-90 km away from Wuhan, who may be second- or third-generation cases by human-to-human transmission of SARS-CoV-2.

In our cohort, 33\% (16) of patients were deceased, and the mortality was lower than that of the first-generation cases reported by Huang et al. in Wuhan, who showed that 38\% ( 5 cases) died in 13 ICU patients with SARS-CoV-2 pneumonia. The median time from admission to critical condition was 4 days, which was longer than that ( 2 days) between hospital admission and ARDS reported by Huang et al. [8]. The deceased patients had shorter median time from admission to critical condition compared with the survivors. These findings suggest that pathogenicity of SARS-CoV-2 seems to decrease with the increase of its generations, and the shorter the time from admission to critical condition, the more serious the illness. Sixty-five percent of the patients were male, and this percentage was also lower than that of ICU patients $(85 \%)$ reported by Huang et al. [8]. The median age of all patients was 63 years, which was older than those reported by Chen et al. (55.5 years) [7], Huang et al. (49 years) [8], Wang et al. (56 years) [9], Liu et al. (57 years) [11], and Zhang et al. (57 years) [12]. In our study, $73 \%$ (36) of patients had comorbid chronic systemic diseases, and hypertension (45\%), chronic heart disease (33\%), diabetes (22\%), and chronic obstructive pulmonary disease (22\%) were the most common comorbidities, whose percentages were higher than those of other reports $[7-9,11,12]$. These discrepancies may be due to $100 \%$ of critical COVID-19 patients in our series. Importantly, we found that the percentage of two or more of comorbid chronic systemic diseases in the deceased patients was higher than that in the survivors, which was consistent with the report by Guan et al., who have verified the significantly escalated risk of poor prognosis in patients with two or more comorbidities as compared with those who had no or only a single comorbidity [13].

In the present study, the most common symptoms included fever (82\%), cough (76\%), fatigue (57\%), and chest tightness/ dyspnea (45\%), which was in accordance with the previous reports [10-15]. Moreover, it was noteworthy that the incidence of gastrointestinal symptoms was $47 \%$ in our cohort, including nausea or vomiting (31\%), diarrhea (22\%), anorexia $(16 \%)$, and abdominal pain (6\%), and gastrointestinal symptoms were more common in the deceased patients than in the survivors. Consistent with our result, Zhang et al. showed that gastrointestinal symptoms were observed in $39.6 \%$ of the patients, and $42.1 \%$ of the severe patients with COVID-19 [12]; Wang et al. found that more than $60 \%$ of the patients with COVID-19 had gastrointestinal symptoms, and the gastrointestinal symptoms were more common in the ICU patients than those in the non-ICU patients [9]; of patients with COVID-19 with gastrointestinal symptoms, $30 \%$ had severe/ critical types, significantly higher than those without gastrointestinal symptoms (8\%) [16]. These results suggest that 
Table 5 Comparison of outcomes between 11 diabetic and 38 nondiabetic critically ill COVID patients

\begin{tabular}{|c|c|c|c|}
\hline Items & Diabetic $(n=11)$ & Nondiabetic $(n=38)$ & $p$ value \\
\hline Age, years & $58(51-62)$ & $68(53-75)$ & 0.116 \\
\hline \multicolumn{4}{|l|}{ Sex, $n(\%)$} \\
\hline Female & $4(36)$ & $13(34)$ & 1.000 \\
\hline Male & $7(64)$ & $25(66)$ & 1.000 \\
\hline \multicolumn{4}{|l|}{ Blood routine } \\
\hline Neutrophil percentage, (\%) (normal range 40-75) & $85.8(71.5-91.5)$ & $73.8(64.2-85.6)$ & 0.045 \\
\hline Lymphocytes $\left(\times 10^{9} / \mathrm{L}\right.$; normal range $\left.1.1-3.2\right)$ & $0.7(0.5-0.9)$ & $0.9(0.7-1.4)$ & 0.168 \\
\hline Lymphocyte percentage, $(\%)$ (normal range $20-50$ ) & $8.5(3.7-19.7)$ & $16.9(9.6-26.1)$ & 0.042 \\
\hline \multicolumn{4}{|l|}{ Infection biomarkers } \\
\hline C-reactive protein (mg/L; normal range $0.0-8.0$ ) & $27.8(12.9-40.8)$ & $40.7(16.6-65.4)$ & 0.297 \\
\hline Procalcitonin (ng/mL; normal range $0.0-0.5)$ & $4.1(0.2-7.9)$ & $0.5(0.1-1.6)$ & 0.106 \\
\hline \multicolumn{4}{|l|}{ Blood biochemistry } \\
\hline Fasting blood glucose (mmol/L; normal range 3.9-6.1) & $10.7(7.4-14.5)$ & $6.1(5.5-8.1)$ & 0.001 \\
\hline Lactate dehydrogenase (U/L; normal range 109-245) & $419.0(326.0-523.8)$ & $268.3(203.3-431.2)$ & 0.042 \\
\hline \multicolumn{4}{|l|}{ Prognosis } \\
\hline Discharge, $n(\%)$ & $6(55)$ & $27(71)$ & 0.466 \\
\hline Death, $n(\%)$ & $5(45)$ & $11(29)$ & 0.466 \\
\hline
\end{tabular}

Data are shown as median (IQR) or $n(\%)$. $p$ values comparing diabetic and nondiabetic COVID patients are from $\chi^{2}$ test, Fisher's exact test, or MannWhitney $U$ test. COVID-19, coronavirus disease 2019; IQR, interquartile range

gastrointestinal symptoms are a potential indicator for severity of COVID-19.

In our cohort, abnormal blood routine results mainly included decreased lymphocytes and lymphocyte percentage, and increased neutrophil percentage. Noticeably, abnormalities of the above parameters were more prominent in the deceased patients than those in the survivors. Infection biomarkers showed that increased level of CRP and PCT was found in $86 \%$ and $55 \%$ of the patients, respectively, and the level of CRP and PCT was higher in the deceased patients compared with that in the survivors. These changes of CRP and PCT may represent more prominent inflammation, whereas higher neutrophil percentage and PCT may be due to more significant secondary bacterial infection in the deceased patients. Numerous studies have shown that lymphopenia is common in patients with COVID-19 [7-12, 14]. Huang et al. showed that lymphopenia was more prominent in the ICU patients than that in the non-ICU patients [8]; Zhang et al.

Table 6 Multivariable logistic regression analysis

\begin{tabular}{lccc}
\hline Items & $p$ value & OR & $95 \% \mathrm{CI}$ \\
\hline Gastrointestinal symptoms $(\geq 1$ symptoms $)$ & 0.009 & 13.4 & $1.9-94.8$ \\
Lymphocytes $(<1.1 \times 109 / \mathrm{L})$ & 0.020 & 25.5 & $1.6-394$ \\
Fasting blood glucose $(\geq 7.0 \mathrm{mmol} / \mathrm{L})$ & 0.016 & 11.7 & $1.6-85.5$ \\
\hline
\end{tabular}

reported that lymphocyte percentage was lower in severe patients compared with that in nonsevere patients [12].

Increased neutrophil has been rarely reported in patients with COVID-19. Huang et al. showed that the median neutrophil count was significantly higher in the ICU patients than that in the non-ICU patients [8]; Chen et al. indicated that increased neutrophils were found in $38 \%$ of the patients with COVID-19 [7]. Increased level of CRP is often reported in recent studies about COVID-19 [7, 11, 12], whereas increased PCT is rarely observed. Zhang et al. [12] reported CRP and PCT concentrations were significantly higher in severe patients with COVID-19 compared with those in nonsevere patients with COVID-19; Chen et al. found that PCT level > $0.5 \mathrm{ng} / \mathrm{mL}$ was one of the independent risk factors associated with fatal outcome in patients with COVID-19 [14]. The above findings were roughly consistent with our results.

Significant abnormal blood biochemistry findings were increased FBG $\geq 7.0 \mathrm{mmol} / \mathrm{L}$, decreased albumin, and increased LDH levels, whereas other liver functions, myocardial zymogram, and renal function indexes were within the normal range in most of the patients. In addition, the level of FBG and LDH was higher in the deceased patients than that in the survivors. Increased LDH level is common in patients with COVID-19 [7-10, 17]. Two reports indicated that LDH level was higher in the ICU patients than that in the non-ICU patients [8, 9]. Few studies have reported hyperglycemia in patients with SARS-CoV-2 infection. Chen et al. showed that increased FBG was found in 52\% of the patients with 2019- 
nCoV infection [7]; Li et al. reported that hyperglycemia (> $7.1 \mathrm{mmol} / \mathrm{L}$ under fasting state) was detected in $56.5 \%(13 / 23)$ of severe cases with COVID-19 and $21.4 \%$ (9/42) of mild cases with COVID-19 [18]. The above results suggest that higher FBG and LDH levels are the important laboratory indexes for patients with COVID-19.

Moreover, in this cohort of 49 critically ill patients with COVID-19, we found that the neutrophil percentage and levels of FBG and LDH were higher and the lymphocyte percentage was lower in the patients with T2DM than those in the patients without T2DM, suggesting the more significant secondary bacterial infection and more severe damages of the lymphocytes, islet cells, and heart function in diabetic critically ill COVID patients.

In our cohort of 49 critically ill patients with COVID-19, all patients showed bilateral viral pneumonia in chest CT images, and there were no significant differences in treatment regimens between the deceased patients and the survivors, including antiviral treatment, corticosteroid treatment, antibacterial treatment, antifungal treatment, respiratory support, and traditional Chinese medicine, which were consistent with the other reports [7-12].

In our study, some independent risk factors for fatal outcome were found by using a multivariable logistic regression analysis. Gastrointestinal symptoms, decreased lymphocytes, and increased FBG were the independent predictive factors for death of critically ill patients with COVID-19.

The multi-organ nature of COVID-19 has been demonstrated in a latest autopsy study [19]. The sequence of SARS-CoV-2 receptor-binding domain is similar to SARS$\mathrm{CoV}$, and angiotensin-converting enzyme 2 (ACE2) is its receptor. ACE2 is highly expressed not only in the lung but also in other organs including the heart, the kidney, and the gastrointestinal tract [20-25]. SARS-CoV-2 may mediate the invasion into gastrointestinal epithelium cells by binding to ACE2 receptor, leading to malabsorption, unbalanced intestinal secretion, and activated enteric nervous system, in turn resulting in gastrointestinal symptoms and electrolyte disturbance $[16,20,22]$. In this way, the patients with gastrointestinal symptoms trend towards the critical type of the disease and a poor prognosis [16].

SARS-CoV-2 might mainly act on lymphocytes, especially $\mathrm{T}$ lymphocytes like SARS-CoV. The virus induces a cytokine storm, generates a series of immune responses, and consumes many immune cells that result in the decrease in lymphocytes and cellular immune deficiency [7, 9]. Damage to T lymphocytes might be an important factor leading to exacerbations of the patients [26].

Hyperglycemia caused by respiratory pathogenic virus infection has been reported. SARS-CoV causes acute pancreatic islet injury by binding to ACE2 receptor, resulting in hyperglycemia in patients with SARS-CoV infection [27]; the report by Wang et al. indicated that high FBG is an independent predictor for severity of H1N1 pneumonia [28]. Therefore, it is reasonable to think that SARS-CoV-2 may invade islet cells through ACE2 receptor and causes hyperglycemia. Increased glucose level inhibits T lymphocyte proliferation, which aggravates lymphopenia and cellular immune dysfunction leading to the deterioration of the disease [29,30]. Indeed, Jin et al. showed that increased glucose level was the independent risk factor for severe/critical COVID-19 in patients with gastrointestinal symptoms [16], which is similar to our result. Thus, high attention should be paid to rescue this process to prevent the further deterioration of COVID-19.

Our study has several limitations. First, the sample size of this study is small. Second, although the risk factors for death of critically ill patients with COVID-19 were identified according to the data on admission, there is still a lack of a predictive model for disease progression. Third, cytokine storm is found in the disease [8]; thus, it would be better if cytokine changes were detected in this study.

\section{Conclusion}

In this study, we reported for the first time that critically ill COVID patients with T2DM had more severe damages of the lymphocytes, islet cells, and heart function, and gastrointestinal symptoms, decreased lymphocytes, and increased FBG are the independent risk factors for death of critically ill patients with COVID-19. The early identification of these risk factors is urgently necessary to facilitate appropriate intensive care.

Acknowledgments The authors appreciate the patients, study investigators, and staff who participated in this study.

Authors' contributions Xiaojuan Peng, Yanfang Chen, Liangfei Deng, Qi Liu, and Qing Li contributed equally to this paper. Xiaojuan Peng, Yanfang Chen, and Qing Li helped design the study, analyzed the study data, helped draft the manuscript, made critical revisions of the manuscript, and provided final approval of the version. Liangfei Deng and Qi Liu helped design the study; acquired epidemiological, demographic, clinical, and laboratory data; analyzed the study data; made critical revisions of the manuscript; and provided final approval of the version. Jie Xiong and Ying Shi analyzed the study data, helped draft the manuscript, and provided final approval of the version. Shaohui Tang designed the study, analyzed study data, drafted the manuscript, made critical revisions of the manuscript, and provided final approval of the version.

Funding This study was supported by Science and Technology Funding Project of Chenzhou, Hunan Province, China (No. zdyf201848); Health Commission Funding Project of Hunan Province, China (No. B2019145); Science and Technology Funding Project of Hunan Province, China (No. 2017SK4010); Key Laboratory of Tumor Precision Medicine, Hunan Colleges and University Project (2019-379); Natural Science Foundation of Hunan Province (No. 2016JJ6140); Medical Science and Technology Foundation of Guangdong Province (No. A2018011). 
Data availability Data sharing will be considered only on a collaborative basis with the principal investigators, after evaluation of the proposed study protocol and statistical analysis plan.

\section{Compliance with ethical standards}

Competing interests The authors declare that they have no conflict of interest.

\section{References}

1. Ksiazek TG, Erdman D, Goldsmith CS, Zaki SR, Peret T, Emery S, et al. A novel coronavirus associated with severe acute respiratory syndrome. N Engl J Med. 2003;348(20):1953-66.

2. Kuiken T, Fouchier RA, Schutten M, et al. Newly discovered coronavirus as the primary cause of severe acute respiratory syndrome. Lancet. 2003;362(9380):263-70.

3. de Groot RJ, Baker SC, Baric RS, Brown CS, Drosten C, Enjuanes L, et al. Middle East respiratory syndrome coronavirus (MERS$\mathrm{CoV}$ ): announcement of the Coronavirus Study Group. J Virol. 2013;87(14):7790-2.

4. WHO. Summary of probable SARS cases with onset of illness from 1 November 2002 to 31 July 2003. Dec 31, 2003. www.who.int/ csr/sars/country/table2004 04 21/en/. Accessed 19 Jan 2020.

5. WHO. Middle East respiratory syndrome coronavirus (MERSCoV). November, 2019. www.who.int/emergencies/mers-cov/en/. Accessed 19 Jan 2020.

6. WHO. Clinical management of severe acute respiratory infection when novel coronavirus ( $\mathrm{nCoV}$ ) infection is suspected: interim guidance. Jan 11, 2020. www.who.int/internal-publicationsdetail/ clinicalmanagementofsevereacuterespiratoryinfectionwhennovel coronavirus(ncov)infectionissuspected. Accessed 20 Jan 2020.

7. Chen N, Zhou M, Dong X, Qu J, Gong F, Han Y, et al. Epidemiological and clinical characteristics of 99 cases of 2019 novel coronavirus pneumonia in Wuhan, China: a descriptive study. Lancet. 2020;395(10223):507-13.

8. Huang C, Wang Y, Li X, Ren L, Zhao J, Hu Y, et al. Clinical features of patients infected with 2019 novel coronavirus in Wuhan, China. Lancet. 2020;395(10223):497-506.

9. Wang $\mathrm{D}, \mathrm{Hu} \mathrm{B}, \mathrm{Hu} \mathrm{C}, \mathrm{Zhu} \mathrm{F}$, Liu X, Zhang J, et al. Clinical characteristics of 138 hospitalized patients with 2019 novel coronavirus-infected pneumonia in Wuhan, China [published online ahead of print, 2020 Feb 7]. JAMA. 2020;323(11):1061-9.

10. Guan WJ, Ni ZY, Hu Y, Liang WH, Ou CQ, He JX, et al. Clinical characteristics of coronavirus disease 2019 in China. N Engl J Med. 2020;382(18): 1708-20.

11. Liu K, Fang YY, Deng Y, Liu W, Wang MF, Ma JP, et al. Clinical characteristics of novel coronavirus cases in tertiary hospitals in Hubei Province. Chin Med J. 2020;133(9):1025-31.

12. Zhang JJ, Dong X, Cao YY, Yuan YD, Yang YB, Yan YQ, et al. Clinical characteristics of 140 patients infected with SARS-CoV-2 in Wuhan, China [published online ahead of print, $2020 \mathrm{Feb} 19]$. Allergy. 2020;75:1730-41. https://doi.org/10.1111/all.14238.

13. Guan WJ, Liang WH, Zhao Y, et al. Comorbidity and its impact on 1590 patients with COVID-19 in China: a nationwide analysis. Eur Respir J. 2020;55(5):2000547 Published 2020 May 14.

14. Chen R, Liang W, Jiang M et al. Risk factors of fatal outcome in hospitalized subjects with coronavirus disease 2019 from a nationwide analysis in China [published online ahead of print, 2020 Apr 15]. Chest. 2020;S0012-3692(20)30710-8.
15. Liang WH, Guan WJ, Li CC et al. Clinical characteristics and outcomes of hospitalised patients with COVID-19 treated in Hubei (epicenter) and outside Hubei (non-epicenter): a nationwide analysis of China [published online ahead of print, 2020 Apr 8]. Eur Respir J. 2020;2000562.

16. Jin X, Lian JS, Hu JH, Gao J, Zheng L, Zhang YM, et al. Epidemiological, clinical and virological characteristics of 74 cases of coronavirus-infected disease 2019 (COVID-19) with gastrointestinal symptoms. Gut. 2020;69(6):1002-9.

17. Liu Y, Yang Y, Zhang C, Huang F, Wang F, Yuan J, et al. Clinical and biochemical indexes from 2019-nCoV infected patients linked to viral loads and lung injury. Sci China Life Sci. 2020;63(3):364 74.

18. Li Z, Liu G, Wang L, Liang Y, Zhou Q, Wu F, et al. From the insight of glucose metabolism disorder: oxygen therapy and blood glucose monitoring are crucial for quarantined COVID-19 patients. Ecotoxicol Environ Saf. 2020;197:110614.

19. Yao XH, Li TY, He ZC, Ping YF, Liu HW, Yu SC, et al. A pathological report of three COVID-19 cases by minimally invasive autopsies. Zhonghua Bing Li Xue Za Zhi. 2020;49(5):411-7.

20. Cheung KS, Hung IFN, Chan PPY, Lung KC, Tso E, Liu R, et al. Gastrointestinal Manifestations of SARS-CoV-2 Infection and Virus Load in Fecal Samples From a Hong Kong Cohort: Systematic Review and Meta-analysis. Gastroenterology. 2020;159(1):81-95.

21. Li LY, Wu W, Chen S, Gu JW, Li XL, Song HJ, et al. Digestive system involvement of novel coronavirus infection: prevention and control infection from a gastroenterology perspective. J Dig Dis. 2020;21(4):199-204.

22. Liang W, Feng Z, Rao S, Xiao C, Xue X, Lin Z, et al. Diarrhoea may be underestimated: a missing link in 2019 novel coronavirus. Gut. 2020;69(6):1141-3.

23. Xiao F, Tang M, Zheng X, Liu Y, Li X, Shan H. Evidence for gastrointestinal infection of SARS-CoV-2. Gastroenterology. 2020;158(6):1831-1833.e3.

24. Harmer D, Gilbert M, Borman R, Clark KL. Quantitative mRNA expression profiling of ACE 2, a novel homologue of angiotensin converting enzyme. FEBS Lett. 2002;532(1-2):107-10.

25. Zhang H, Li HB, Lyu JR, Lei XM, Li W, Wu G, et al. Specific ACE2 expression in small intestinal enterocytes may cause gastrointestinal symptoms and injury after 2019-nCoV infection [published online ahead of print, 2020 Apr 18]. Int J Infect Dis. 2020;96:19-24.

26. Liu WJ, Zhao M, Liu K, Xu K, Wong G, Tan W, et al. T-cell immunity of SARS-CoV: implications for vaccine development against MERS-CoV. Antivir Res. 2017;137:82-92.

27. Yang JK, Lin SS, Ji XJ, Guo LM. Binding of SARS coronavirus to its receptor damages islets and causes acute diabetes. Acta Diabetol. 2010;47(3):193-9.

28. Wang W, Chen H, Li Q, Qiu B, Wang J, Sun X, et al. Fasting plasma glucose is an independent predictor for severity of H1N1 pneumonia. BMC Infect Dis. 2011;11:104.

29. Price CL, Hassi HO, English NR, Blakemore AI, Stagg AJ, Knight SC. Methylglyoxal modulates immune responses: relevance to diabetes. J Cell Mol Med. 2010;14(6B):1806-15.

30. Klekotka RB, Mizgała E, Król W. The etiology of lower respiratory tract infections in people with diabetes. Pneumonol Alergol Pol. 2015;83(5):401-8.

Publisher's note Springer Nature remains neutral with regard to jurisdictional claims in published maps and institutional affiliations. 